\title{
Polyamine Metabolism during the Perinatal Development of the Rabbit Right and Left Ventricle
}

\author{
ROBERT J. BOUCEK, JR, ${ }^{(28)}$ with the Technical Assistance of ROWENA DAVIDSON
}

Department of Pediatrics and Biochemistry, Vanderbilt Medical Center, Nashville, Tennessee, USA

\section{Summary}

The right ventricular (RV) and left ventricular ( $L V)$ free wall weights, ornithine decarboxylase (ODC) specific activity and polyamine content were determined in fetal, 1-, 2-, 7-, 14-, and 21 day-old rabbit hearts. There was a significant increase in the $\mathrm{LV}$ free wall weight and a decrease in $R V$ free wall weight between 1-7 days. By day 7 the LV/RV mass ratio doubled and reached a ratio comparable to that seen in adult rabbit hearts. The rate of change in the $L V$ and $R V$ free wall weights were comparable after day 7 .

There was a significant increase in LV ODC specific activity and a decrease in RV ODC specific activity after birth. The LV ODC specific activity was significantly greater than RV ODC specific activity in both 1 and 2 day old rabbit hearts whereas they were not significantly different in 7 and 14 day old rabbit hearts.

The molar content (nmoles/mg wet weight or nmoles/mg protein) of putrescine decreased approximately 3-fold fter birth in the RV but increased approximately 2 -fold in the $\mathrm{LV}$. The molar content of spermidine and spermine was transiently increased after birth (1 day old) in both RV and LV with approximately a 2fold increase in spermidine and a 2.5 -fold ( $\mathrm{LV}$ ) and 4-fold (RV) increase in spermine content. The increase in $\mathrm{LV}$ putrescine content after birth was due, at least in part, to the observed increase in ODC specific activity in the LV free wall. The putrescine/spermidine ratio increased in the $L V$ and decreased in the RV immediately after birth up to day 7. As opposed to the shortlived increase in spermidine and spermine observed in both the $R V$ and the LV free wall, increased ODC specific activity and putrescine accumulation uniquely characterized the preferential growth of the LV between day 1 and 7.

\section{Speculation}

The postnatally developing heart affords an ideal model for studying the relationship between pressure loading and adaptive myocardial growth because paired comparisons of left and right ventricular parameters are possible. Age-related changes in left ventricular (LV) growth, ornithine decarboxylase (ODC) specific activity and putrescine accumulation occur immediately after birth and were clearly different from the right ventricle. This increase in $L V O D C$ activity and putrescine synthesis may be secondary to the alterations in hemodynamic loading of the $L V$ in the newborn. If cellular hyperplasia accounts for this preferential growth of the $\mathrm{LV}$ in the developing rabbit heart, as has been reported to be the case in the developing rat heart, it is speculated that polyamine metabolism may play a role in myocardial cellular hyperplasia.

Mammalian myocardial growth after birth is characterized by overall growth of the heart with disproportionatly greater growth of the left (or systemic) ventricle (LV) compared with the right (or pulmonic) ventricle (RV) $(12,18)$. The rapid growth of the LV in response to the recognized postnatal hemodynamic and structural cardiovascular alterations (19) is accompanied by increased myocardial cell number as well as increased cell size (10). In the first wk of postnatal life an increase in myocyte number accounts for the 2-fold weight difference between the left and right ventricles for developing rats (2).

Other differences also have been observed in the rate of microvascular and subcellular organelle maturation of the left versus right ventricle $(16,23)$. From the morphologic features of the neonatal rat heart, $\mathrm{LV}$ growth is analogous to combined eccentric and concentric hypertrophy whereas right ventricular growth is analagous to eccentric hypertrophy (2). Presumably the postnatal pressure loading of the newborn $\mathrm{LV}$ accelerates its rate of growth with features similar to concentric hypertrophy due to pressure overloading in pathologic states. Thus the postnatally developing heart affords an ideal model for studying the relationship between pressure loading and adaptive myocardial growth because paired comparisons of left and right ventricular parameters are possible.

An increase in ornithine decarboxylase (EC. 4.1.1.17) activity, and increased polyamine production by this enzyme, occurs during the initiation of adaptive myocardial growth in the pressure overloaded adult heart. Acute banding experiments have demonstrated an early rise in ornithine decarboxylase (ODC) activity and an increase in polyamine content in the right ventricle following pulmonary artery banding (20) and the left ventricle following aortic banding $(4,7)$. This increase in ODC activity and polyamine synthesis has been suggested to regulate myocardial growth by the regulation of: (1) the synthesis of RNA $(4,5) ;(2)$ the translation of mRNA (9); or (3) gene expression by the polyamine regulation of the acetylation of nucleohistones $(6,15)$. Recently, ODC activity and polyamine content have been shown to be increased in the thyroxine-induced hypertrophy of the adult rat heart (17). With the use of 1,3-diamino-2-propanol, a reported inhibitor of ODC activity, the increase in polyamine content did not appear to be essential for hypertrophy in this model and species (17). This report does speculate that the hypertrophy of myocardial cells, as opposed to hyperplasia, may not require enhanced polyamine synthesis (17). Thus the cumulative evidence to date suggests that increased production of the polyamines and ornithine decarboxylase activity are associated with cardiac hypertrophy but their role is still unclear.

Because of the potential role of the polyamines in the regulation of cellular hyperplasia (26) the age-related changes in ODC activity and polyamine content were investigated in the left and right ventricles of the developing rabbit heart. Because the preferential growth of the LV after birth is due to a higher rate of cellular proliferation than the RV (2), the relationship between polyamine metabolism and myocardial hyperplasia may be examined by comparing polyamine synthesis in the two ventricles. The goal of this study was to determine if the initiation of the physiologic growth of the $\mathrm{LV}$ was related to an increase in ODC activity and polyamine accumulation compared with the RV. An additional goal was to determine if there was a characteristic polyamine profile in the ventricle undergoing a growth response 
to increased pressure loading analagous to that seen in pressureoverloaded hypertrophy models (20).

\section{MATERIALS AND METHODS}

Animal protocol. White New Zealand rabbits were housed and fed standard Purina rabbit chow and water ad libitum. Age-related changes in body, heart, RV and LV weights, ODC specific activity and polyamine content was determined in rabbits $1,2,7,14$ and 21-days after delivery. Similar determinations were also made in fetal rabbits from a litter removed surgically from a pregnant doe at approximately 1 day before expected partuition. All other agerelated comparisons of body and heart weights, LV and RV weights, ODC specific activity and polyamine content were made between animals of at least two separate and comparably sized litters. This protocol was adopted because removing litter mates at different ages has been shown to accelerate the rate of growth of the remaining animals in the litter (10). All differences between right and left ventricular free wall weights, ODC specific activity, and polyamines content at each of the age points were analyzed as paired observations. Litters with less than five animals were not used.

Tissue preparation. After rapid cervical fracture, the heart was quickly dissected from the animal, rinsed with chilled saline to remove all blood, blotted and weighed. The LV and RV free walls, including the papillary muscles, were dissected free, rinsed, blotted and weighed on an analytical balance (Mettler).

The RV and LV free walls were submerged in buffer at $4^{\circ} \mathrm{C}$ containing: Tris-HCl (25 mM, pH 7.4); dithiothreitol (5.0 mM); and pyridoxal-5'-phosphate $(0.25 \mathrm{mM})$. The ratio of buffer volume to tissue volume was 3.0 assuming a tissue density of $1 \mathrm{~g} / \mathrm{ml}$. Before mechanical homogenization the muscle and buffer were frozen in dry ice/isopropanol slurry and thawed to improve cellular disruption and inactivate a "non-specific" decarboxylase activity present under certain conditions in the homogenate (personal observation). Mechanical homogenization was carried out at $4^{\circ} \mathrm{C}$ with a Potter-Elvehjem glass homogenizer (approximately 10 strokes).

$O D C$ activity assay. ODC activity was assayed in the supernatant fraction of the homogenate $\left(12,000 \times \mathrm{g}\right.$ for $5^{\prime}$; Eppendorf micro-centrifuge). The assay medium contained: Tris- $\mathrm{HCl}(23$ $\mathrm{mM}$, pH 7.4); dithiothreitol (4.5 mM); and pyridoxal-5'-phosphate $(0.23 \mathrm{mM})$ and a final volume of $0.120 \mathrm{ml}$. Incubation was initiated by the addition of substrate $\left[\left(\mathrm{L}-\left(1-{ }^{14} \mathrm{C}\right)\right.\right.$-ornithine; specific activity of $4.17 \mu \mathrm{C} / \mu \mathrm{mole}$ and an assay ornithine concentration of $1 \mathrm{mM}$ ] and quickly warming to $37^{\circ} \mathrm{C}$. The $\mathrm{CO}_{2}$ was collected in a center well containing NCS as previously described (24). After $40 \mathrm{sec}$ the assay was stopped with chilled $10 \%$ trichloroacetic acid (TCA) and the percipitable protein determined (13). The final substrate concentration of $1 \mathrm{mM}$ was at least twice the apparent $\mathrm{Km}$ for rabbit myocardial ODC. The center well containing evolved $\left[{ }^{14} \mathrm{CO}_{2}\right]$ was immersed in $10 \mathrm{ml}$ of Econofluor and counted at $4{ }^{\circ} \mathrm{C}$ in a liquid scintillation spectrometer (Beckman Model \#LS 250).

All assays were performed in duplicate. Counting efficiency exceeded $85 \%$ using the external standard method to estimate sample quenching. Nonenzymatic decarboxylation was estimated with each determination in a comparable aliquot of an aciddenatured supernatant, and was subtracted from the total rate of decarboxylation. ODC specific activity was expressed as pmoles $\mathrm{CO}_{2}$ per $\mathrm{mg}$ of TCA percipitable protein per h at $37^{\circ} \mathrm{C}$.

Polyamine determinations. Perchloric acid extractable (non-hydrolyzed) putrescine, spermidine and spermine content in mechanically disrupted ventricular free walls were determined using the high performance liquid chromatographic method of Seiler et al. (22). The myocardial homogenate was prepared as above except it was acidified by the addition of $0.1 \mathrm{ml}$ of $1 \mathrm{~N}$ hydrochloric acid to ensure the stabilization of the polyamines as salts. One to ten nmoles of 1,6-diaminohexane was added to the homogenate as an internal standard to monitor and correct for differences in sample recovery which generally exceeded $80 \%$. The $\mathrm{HCl}$-acidified myocardial homogenate was frozen with dry ice/isopropanol and lyophilized to dryness. After lyophilization, $0.1 \mathrm{ml}$ of $0.2 \mathrm{~N}$ perchloric acid was added to the lyophilized homogenate and perchloric acid insoluble material removed by centrifugation. The polyamines extractable by perchloric acid were alkalinized with sodium carbonate in light-shielded tubes, and $0.3 \mathrm{ml}$ of a freshly prepared $10 \mathrm{mg} / \mathrm{ml}$ dansyl chloride solution in acetone (Aldreich Co.) was added. After an overnight reaction at room temperature, unreacted dansyl chloride was removed by the addition of proline. The dansyl derivatives of the polyamines were extracted with toluene $(3 \mathrm{ml} \times 2)$. A preseparation of the polyamine derivatives from other reaction products was performed (22) before high performance liquid chromotgraphy. Approximately $80-90 \%$ of the internal standard and labeled polyamines could be dansylated and recovered by this method (personal observation). A filter spectrophotofluorometer (Fluoro-Monitor, Aminco Co.) with a Corning 7-60 primary filter $(355 \mathrm{~nm})$, and a Wratten $2 \mathrm{~A}-12$ secondary filter $(510 \mathrm{~nm})$ was used to detect the fluorescence of the dansyl derivatives eluted with a non-linear gradient between $45-100 \%$ methanol.

Relative fluorescence of the dansyl derivatives in samples of authentic standards dansylated under identical conditions was determined with each group of myocardial samples. Identification of the putrescine, spermidine and spermine derivatives was based on the comigration with dansyl derivatives of authentic standards. Each determination was corrected for internal standard recovery.

Statistical design. Statistical analysis of the difference in wet weight, protein and polyamine content, and ODC specific activity between the RV and $\mathrm{LV}$ was determined using a paired $t$ test for each age group. The level of significance chosen was $P<0.05$. Using linear regression analysis of the fluorescent peak areas of authentic polyamine standards betweeen 100 and 1000 pmoles, the molar content of putrescine, spermidine, and spermine were calculated in the right and left ventricles of the rabbit hearts. The results were expressed as the molar amount of the polyamines both per milligram protein and per milligram wet weight. Statistical analysis of the difference in polyamine content at different ages in either the right and left ventricle was made using an unpaired $t$ test and Student's distribution.

\section{RESULTS}

Whole body weight increased in a linear fashion $(r=0.989)$ from day 2 to day 21 at a rate of nearly $16 \mathrm{~g}$ per day (Fig. l). The growth rate of the whole heart and the body weight were parallel up to 14 days (Fig. 1) but heart growth slowed after that time. At 21 days the heart weight was approximately $0.37 \%$ of the body weight whereas at 7 and 14 days the heart weight was approximately $0.42 \%$ and $0.49 \%$ respectively, of the body weight.

There was a significant increase in the weight of the free wall of left ventricle (LV) from $92.5 \pm 9.0 \mathrm{mg}$ to $182 \pm 9.1 \mathrm{mg}$ (mean \pm S.E.; $P<0.01$ ) between $1-7$ days (Fig. 1), in contrast $\mathrm{RV}$ free wall weight decreased from day 1 to day $2(106.6 \pm 7$ to $84.0 \pm 5 \mathrm{mg})$ and then only increased slightly to $93.9 \pm 7 \mathrm{mg}$ at 7 days. The difference between the RV and the LV free wall weights in 1- and 2-day-old rabbits was not statistically significant but by day seven mean LV free wall was significantly greater than the mean RV free wall weight $(P<0.001)$. The $\mathrm{LV} / \mathrm{RV}$ wet weight ratios were 0.87 and 1.16 in $\mathrm{I}-$ and 2 -day-old rabbits, but by day 7 the LV/ RV almost doubled to 1.9. At 14 days and 21 days the LV/RV ratios were 2.1 , and 2.0 respectively suggesting comparable rates of growth of the two ventricles after day 7.

The sarcoplasmic (soluble) protein content per mg wet weight was comparable in both LV and RV after day 1 (Fig. 2A). The increase in wet weight of the LV after birth was accompanied by an increase in the LV sarcoplasmic protein content with no significant increase in RV sarcoplasmic protein content until day 21 (Fig. 2B).

The mean LV free wall ODC specific activity at day 1 and 2 was significantly higher than fetal activity $(P<0.01)$ and significantly greater than in the RV free wall in both one and 2-day-old rabbits (Fig. 3). The mean RV free wall ODC specific activity was 


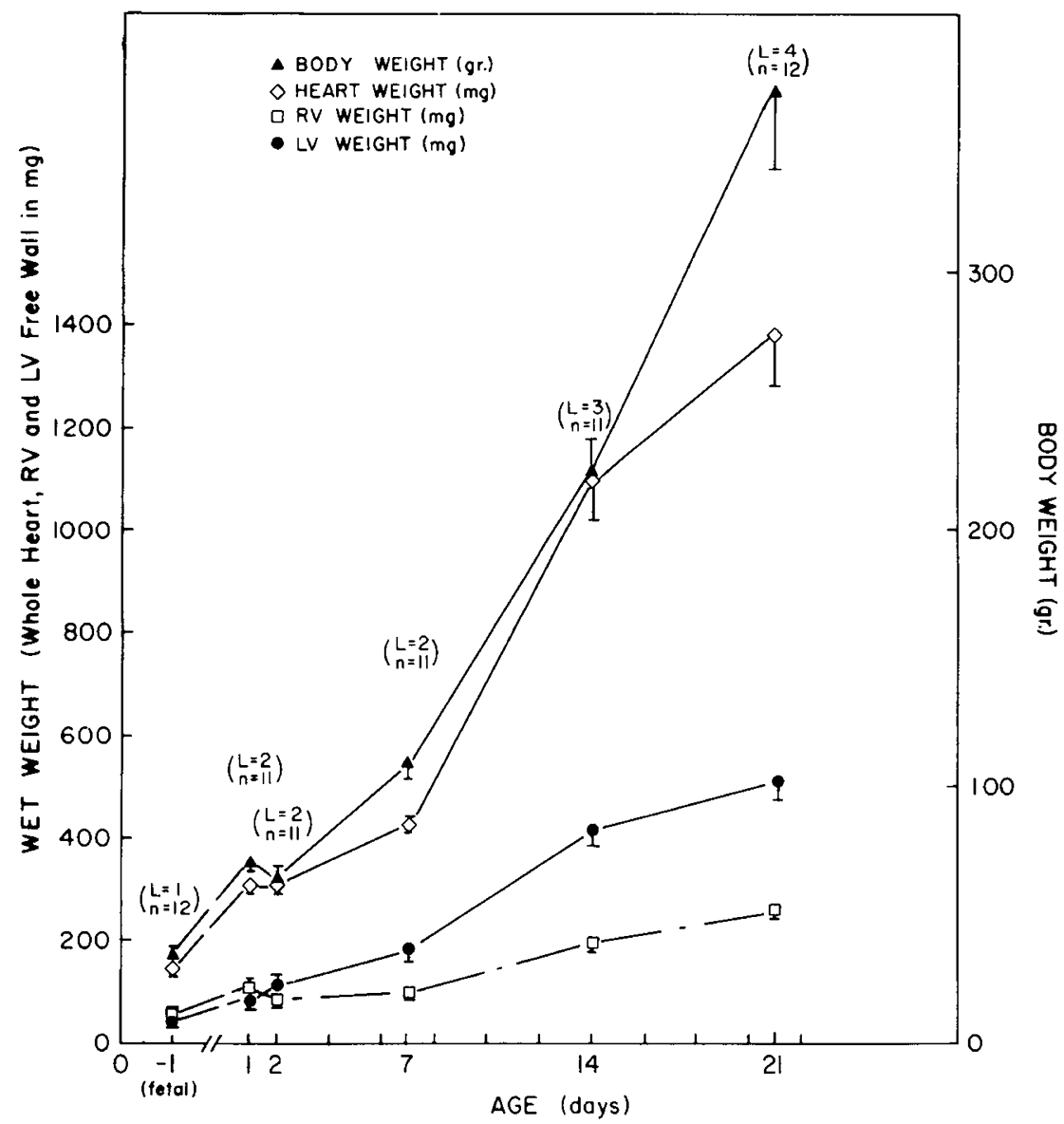

Fig. 1. Age related changes in heart and body weight of the developing rabbit. The mean \pm the S.E. of the wet weights for $(n)$ number of determinations from (L) number of separate litters are shown. The body weight $(\boldsymbol{\Delta}-\mathbf{\Delta})$ was expressed in $\mathrm{g}$ whereas the other weights are expressed in $\mathrm{mg}$. The heart weights $(\diamond \smile)$ included the weights of the atria and intraventricular septum. By paired $t$ test there was no significant difference in left ventricular $(-\circ)$ and right $\left(\begin{array}{ll}\square & \square\end{array}\right)$ ventricular free wall wet weights (in $\mathrm{mg}$ ) until day 7 . After day 7 left ventricule (LV) free wall weights significantly exceeded right ventricle (RV) free wall weights.

significantly decreased in 1-day-old rabbit hearts compared with the fetal values $(P<0.05$ using a single tailed $t$ test of unpaired data). In the free walls of 21-day-old rabbits LV ODC specific activity was slightly less than RV ODC specific activity though this may not have any physiologic relevance in light of the closeness of the values.

After birth there was a transient increase in the RV content of spermidine $(P<0.01$; Fig. 4$)$ and spermine $(P<0.001$; Fig. 5$)$ compared with the fetal RV contents immediately after birth whereas putrescine concentration did not change $(P=$ NS; Fig. 6). After birth there was also a transient increase in the LV content of spermidine $(P<0.01$; Fig. 4$)$, and spermine $(P<0.005$; Fig. 5).

There was a significantly higher content of putrescine in the LV of both 1- and 2-day-old rabbits compared both with the putrescine content (nmoles/mg protein) in the fetal LV free wall $(P<$ 0.05 ; Fig. 6 ), and the mean putrescine content in the RV free wall. The putrescine accumulation in the LV was most likely due to increased synthesis concomittant with the observed increase in ODC activity in the LV free wall. The LV putrescine content returned to levels comparable to $\mathrm{RV}$ at day 7 . Putrescine content (nmoles/mg wet weight) was also significantly greater in 1- and 2day-old $L V$ free walls compared to $R V$ free walls (data not shown).

The total molar polyamine content of all three polyamines (Fig. 7) increased after birth and was significantly greater in the 1-dayold LV and RV free wall when compared with the fetal LV and RV $(P<0.001)$. The total polyamine content did not differ at any age in LV versus RV. After day 1 the total polyamine content fell and by day 7 was slightly below the respective fetal content. Between 2-7 days the net polyamine synthesis in the LV free wall increased such that during the period when $L V / R V$ weight ratio doubled in the first postnatal wk, the molar content of polyamines per mg protein (Fig. 7) and per mg wet weight (data not shown) was comparable in both LV and RV (Fig. 7).

The molar ratio of putrescine to spermidine was significantly greater in the RV free wall than LV free wall of fetal hearts (Fig. 8); however, after birth this ratio increased in the LV free wall and decreased in the RV free wall and the ratio was significantly greater in LV free walls than in RV free walls of both the 1- and 2-day-old hearts (Fig. 8). After day 2 this ratio was comparable in both free walls with a decreasing ratio with age in both ventricles after day 7. By day 21 the putrescine to spermidine ratio was still slightly higher than the adult rabbit LV $(0.249 \pm 0.03$; mean \pm $\mathrm{SE}, n=8)$ and $\mathrm{RV}(0.201 \pm 0.02$; mean $\pm \mathrm{SE}, n=8)$. No significant difference between $R V$ and $L V$ putrescine to spermidine ratio was found in either 21-day-old or adult rabbits.

\section{DISCUSSION}

The postnatal development of the rat heart has been recently described, from a morphologic perspective, and clearly defines the rapid growth of the LV myocytes after birth (2). During the period of rapid growth of the $\mathrm{LV}$, there is both cell hyperplasia (2) and enlargement requiring the new synthesis of DNA (10), RNA, and particularly new protein (27). In contrast, the postnatal development of the RV is characterized by predominant cell enlargement $(2,10)$. In the rabbit heart there also is preferential LV growth (Fig. 1 and 2). In agreement with previous reports (12) the rabbit RV and LV free wall weights in this study (Fig. 1) were comparable at birth $(\mathrm{LV} / \mathrm{RV}$ weight ratio $=0.87$ at day 1$)$. Up to day 7 

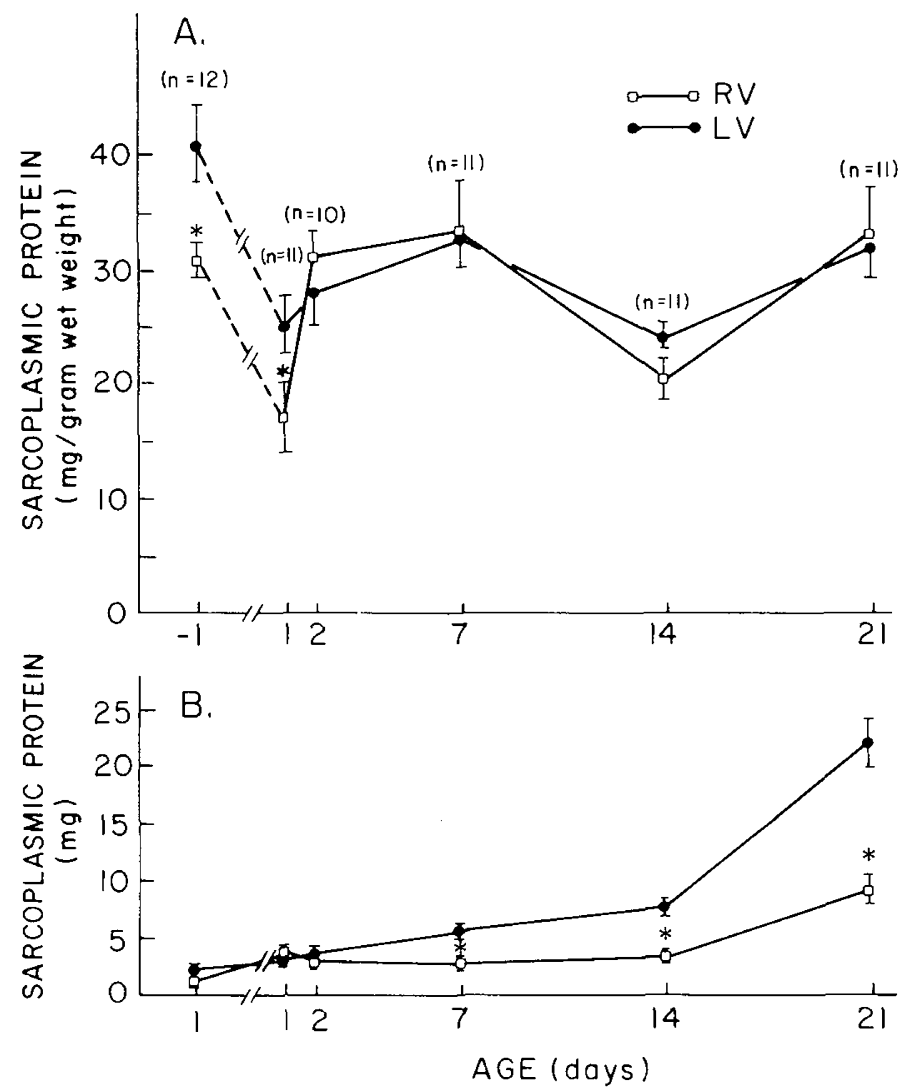

Fig. 2. Age related changes in total sarcoplasmic protein content and sarcoplasmic protein per $g$ wet weight. The right and left ventricular free walls were removed from rabbits at different ages. A $30 \%$ homogenate was prepared as described and a supernatant fraction obtained after centrifugation. The total acid-percipitable protein was determined in the supernatant fraction and defined as the total sarcoplasmic protein. The mean \pm the S.E. sarcoplasmic protein per $\mathrm{mg}$ wet weight $(A)$ and the total sarcoplasmic protein in the right (RV) ( $\square \square \square$ ) and left (- ventricular $(\mathrm{LV})$ free walls were determined and the paired determination at each age analyzed for significance by paired $t$ test. A $P<0.01$ is denoted by $\left({ }^{*}\right)$ for $n$ number of determinations.

the rate of $L V$ free wall growth exceeded the rate of $R V$ growth such that the ratio of LV to RV weight after day 7 was approximately 2 (Fig. 1), also in good agreement with the previous reported ratio of 1.86 in adult rabbit hearts (12). Further studies, such as the careful morphometric analysis of Anversa et al. $(2,16)$ will be necessary to determine if the preferential LV growth in the rabbit reported herewithin is comparable to the preferential wet weight growth due to myocyte hyperplasia in the LV of the rat heart between 1 and 11 days after birth.

It is important to emphasize that in this study the soluble protein content of the respective free walls is reported in order to relate the changes in the enzymatic activity of the soluble enzyme, ODC, with other soluble proteins. Though it was possible that the preferential increase in LV wet weight after birth may reflect a selective increase in the water content of the LV free wall, evidence was presented that makes this unlikely (Fig. 2). LV wet weight (Fig. 1) and the amount of soluble protein (Fig. 2B) increased between day $I$ and 7 whereas in contrast RV wet weight (Fig. 1) and the amount of soluble protein in the RV remained constant (Fig. 2B). Thus the amount of soluble protein per unit of wet weight actually increased slightly after birth in both the LV and the RV becoming equal in the LV and RV at day 2 which would argue against a preferential accumulation of $L V$ water content.

The preferential growth of the LV when compared to the RV that occurs after birth was accompanied by a preferential increase in LV ODC specific activity compared with both the fetal LV, and the RV free wall activity at day 1 and 2 after birth. This is the first reported comparison of LV and RV ODC specific activity during this critical period of post-natal development of the heart. The increase in ODC activity in the LV after birth reported herewithin in the rabbit may account for the reported increase in ODC specific activity in whole heart homogenates in the rat $(1$, 21). This increase in the LV ODC activity at day $l$ and 2 occurred before an increase in soluble protein content in the LV. Thus LV ODC specific activity was increased either by a selective increase

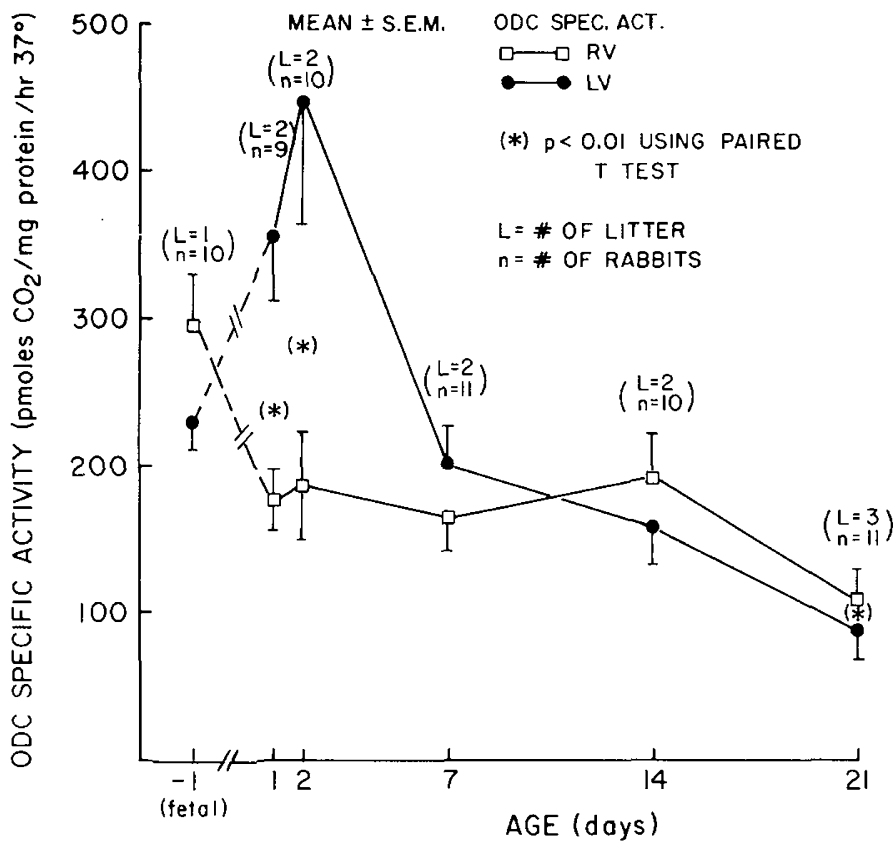

Fig. 3. Age related changes in rabbit myocardial ornithine decarboxylase specific activity. Ornithine decarboxylase activity was determined in the supernatant fraction of myocardial homogenates of right $(\square-\square)$ and left $(-)$ ventricles from rabbits at different ages after birth. The mean and S.E. of the specific activity, at saturating concentrations of substrate and cofactor, was determined and expressed as a function of age. The $\left({ }^{*}\right)$ denotes significance at the $P<0.01$ level by a paired $t$ test analysis of the difference between left (LV) and right ventricle (RV) at any given age.

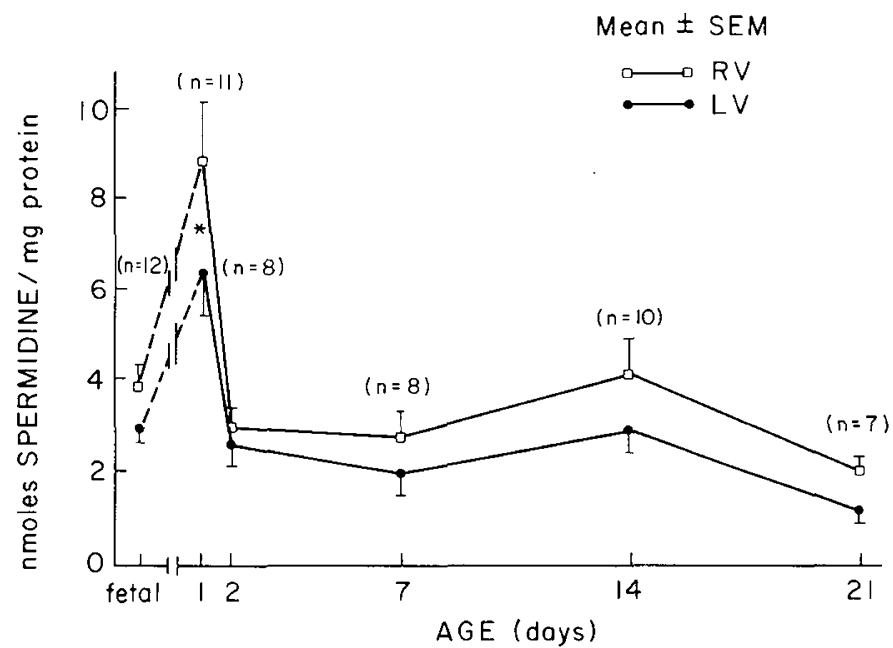

Fig. 4. Age related changes in rabbit myocardial spermidine. The perchloric acid extractable spermidine per $\mathrm{mg}$ protein was determined in the right (RV) ( $\square-\square$ ) and left (- - ventricular (LV) free walls of rabbits at different ages after birth. The mean \pm S.E. of $(n)$ number of paired determinations was expressed as a function of age in this figure. The $\left({ }^{*}\right)$ denotes the significance at the $P<0.01$ level by a paired $t$ test of the difference in spermidine in the $L V$ and RV at any given age. 


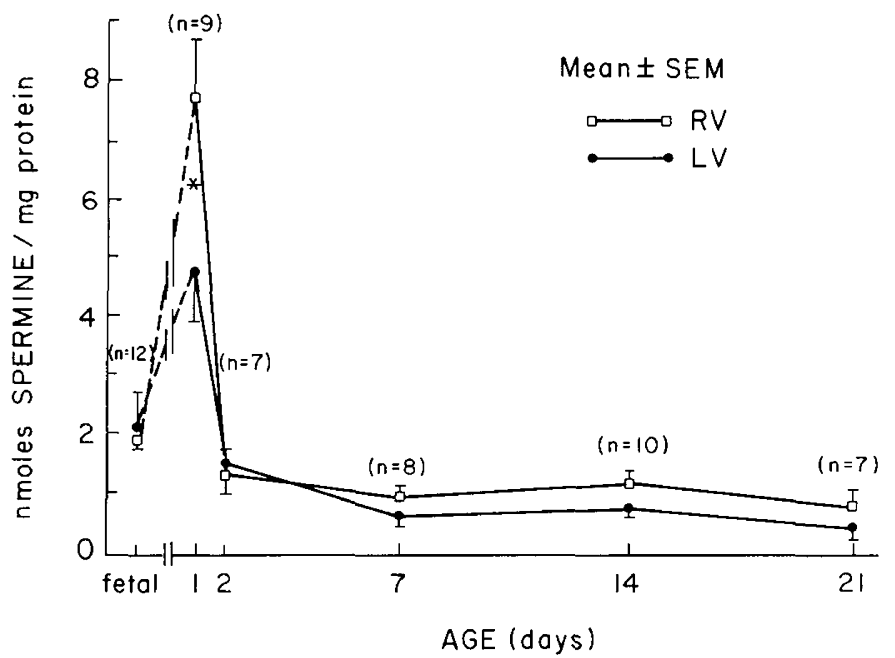

Fig. 5. Age related changes in rabbit myocardial spermine. The perchloric acid extractable spermine per $\mathrm{mg}$ protein was determined in the right (RV) ( $\square-\square$ ) and left (- - ) ventricular (LV) free walls of rabbits at different ages after birth. The mean \pm S.E. of $(n)$ number of paired determinations was expressed as a function of age in this figure. The $\left(^{*}\right)$ denotes significance at the $P<0.01$ level by a paired $t$ test of the difference in spermine in the LV and RV at any given age.

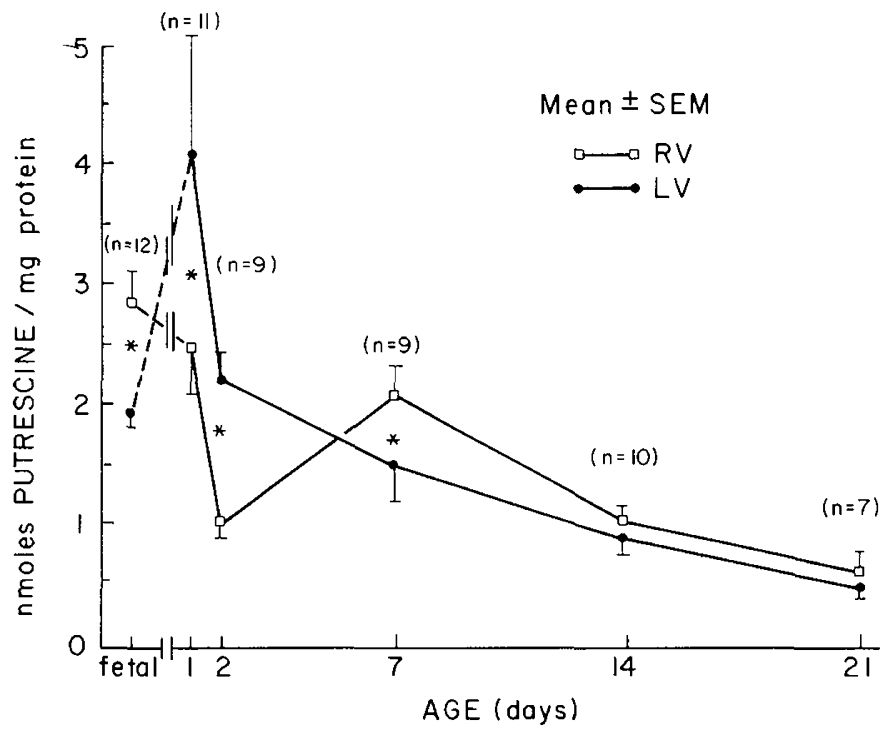

Fig. 6. Age related changes in rabbit myocardial putrescine. The perchloric acid extractable putrescine were determined in the right (RV) $(\square \square \square)$ and left $(\square)$ ventricular (LV) free walls of rabbits at different ages. The mean \pm S.E. of the putrescine content of $(n)$ number of determinations were expressed as a function of age in this figure. The $\left.{ }^{*}\right)$ denotes significance at the $P<0.01$ level by a paired $t$ test analysis of the difference between $L V$ and $R V$ at any given age.

in its activity as postulated by Lou and Slotkin (11) or was a reflection of stimulated protein synthesis with an earlier increase in the accumulation of those proteins with rapid turnover rates such as ODC (17) before a detectable increase in soluble protein content. At this time, it is technically impossible to accurately quantitate the amount of ODC present therefore it's only possible to speculate about the mechanism for the LV specific increase in ODC activity (Fig. 3).

It has been recently suggested that an increase in either circulating and/or endogenous catecholamines may activate ODC in the rat heart $(3,25)$. ODC activity in these reports was determined in the whole heart; however, because this study has shown that RV ODC did not increase at day 1 or 2 it is unlikely that circulating factors such as catecholamines initiate the observed increase in LV ODC specific activity as had been previously suggested $(3,25)$. Neither do endogenous catecholamines appear to rise in the rabbit myocardium until 7 days (8) suggesting that the endogenous release of catecholamines may not induce $\mathrm{LV}$ ODC activity.

The in vivo significance of the observed changes in ODC activity, assayed in vitro (Fig. 3), was supported by the similarities in the direction of change found in the ODC activity, and the myocardial content of the product of ODC, putrescine. As ODC activity per $\mathrm{mg}$ protein increased in the LV immediately after birth there was a concomittant increase in putrescine content per mg protein. Conversely, as RV ODC activity decreased immediately after birth, putrescine content also decreased. As the LV ODC activity also decreased from day 2 to day 7, putrescine

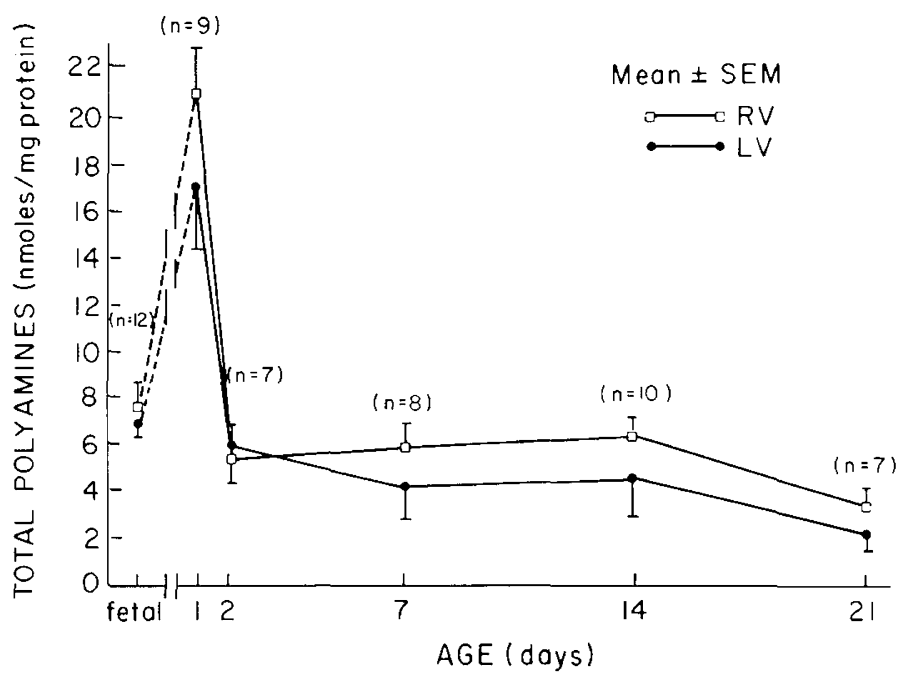

Fig. 7. Age related changes in rabbit myocardial polyamine content. The perchloric extractable total polyamine content was determined in the right (RV) $(\square-\square)$ and left $(-\square)$ ventricular (LV) free walls of rabbit hearts at different ages. The content of putrescine, spermidine, and spermine per $\mathrm{mg}$ protein were individually determined in $(n)$ number of paired ventricular free walls and the mean \pm S.E. expressed as a function of age in days. The $\left(^{*}\right)$ denotes significance at the $P<0.01$ level by a paired $t$ test of the difference between $L V$ and RV at any given age.

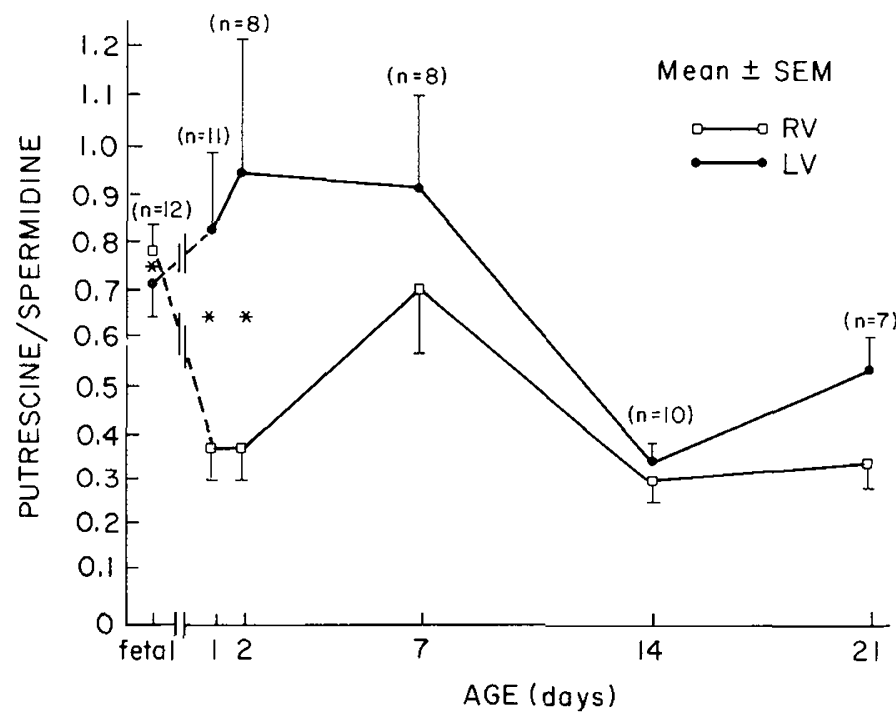

Fig. 8. Age related changes in the putrescine:spermidine ratio. The mean \pm S.E. molar ratios of putrescine to spermidine for $(n)$ number of paired determinations in rabbit right ventricular $(R V)\left(\begin{array}{l}\square- \\ -\end{array}\right)$ and left ventricular (LV) (-) free wall were expressed as a function of age in this figure. The $\left({ }^{*}\right)$ denotes significance at the $P<0.01$ level by paired $t$ test of the difference in the ratios between the LV and RV. 
content also decreased. Therefore, with the exception of day 2 values, changes in putrescine content were generally concordant with the changes in the in vitro ODC activity in the rabbit heart. It is important to emphasize that the rate of putrescine utilization and degradation must also be taken into consideration before it will be possible to establish that myocardial putrescine content is solely determined by changes in ODC activity.

The myocardial polyamine content, as determined by highperformance liquid chromatographic separation and quantitation of the eluted dansyl derivatives, underwent ventricle-specific and age related changes that have not been previously reported. Russell and McVicker (2) previously reported that the polyamine content in the heart increased after birth. Comparison of the polyamine contents in a 22-day-old fetal rat with the 1-day-old newborn rat demonstrated an increase in putrescine content $(0.78$ $\pm 0.08 \mathrm{nmoles} / \mathrm{mg}$ total protein to $0.91 \pm 0.009 \mathrm{nmoles} / \mathrm{mg}$ total protein) and spermidine $(4.60 \pm 0.65 \mathrm{nmoles} / \mathrm{mg}$ protein to 6.0 $\pm 0.68 \mathrm{nmoles} / \mathrm{mg}$ protein) using butanol extraction, high voltage electrophoretic separation, and quantitation by ninhydrin (21).

Comparison of the absolute values for the respective polyamines reported in the newborn rat heart (21) with those determined herewithin in the newborn rabbit is difficult since: (1) the newborn rat polyamine values were determined in pooled whole hearts rather than in the individual ventricles; (2) putrescine by ninhydrin quantitation in the newborn rat (21) may have overestimated putrescine (17); and ( 3 ) there may be species differences and agerelated differences in the direction of change in the polyamines in the two ventricles not previously appreciated in whole heart. For instance, the spermine content is lower than the spermidine content in the rabbit heart (personal observation, 20) and neonatal rat heart (21), whereas the spermine content is higher than the spermidine content in the adult rat heart (17). Despite these difficulties, several points of agreement between values obtained in this laboratory with published reports in adult rabbit RV have strengthened the confidence in the methods and observations reported herewithin. Using the HPLC method described herewithin, the content of spermidine and spermine in the adult rabbit RV were $82.4 \pm 0.4$ and $29.9 \pm 8.8$ nmoles/gram wet weight, respectively, which were comparable $(n=8$; mean \pm S.E., to the spermidine content of $122.5 \pm 13.5(n=2$; mean \pm S.E.) nmoles/ $\mathrm{g}$ and spermine content of $85.8 \pm 13.5 \mathrm{nmoles} / \mathrm{g}$ in the adult rabbit RV reported by Russell et al. using high voltage electrophoretic separation and ninhydrin staining (20).

The putrescine content in the adult rabbit $\mathrm{RV}$ determined in this laboratory was $15.5 \pm 1.8$ compared with $42.5 \pm 7.5$ nmoles/ $\mathrm{g}$ reported by Russell et al. (20). If putrescine content in the adult RV was overestimated with ninhydrin by Russell et al. (20), as has been suggested by Pegg and Hibasami (17), then the putrescine content determined in this laboratory in the rabbit $\mathrm{RV}$ determined by the HPLC method may more accurately reflect putrescine content in the rabbit RV. The fluorometrically quantitated putrescine content of $5.0 \pm 2$ nmoles $/ g$ (mean \pm S.D.) in the whole adult rat heart (17) was lower than observed in this laboratory in the rabbit heart possibly due in part to species differences similar to the differences reported in spermine and spermidine.

Species differences may also explain the discrepancy between the reported putrescine/spermidine ratio of 0.15 in the $1-$ and 2 day-old rat heart (21) and that ratio of approximately 1.0 or 0.3 in $\mathrm{LV}$ and RV respectively of the rabbit heart (Fig. 8); however, it is possible that analysis of this ratio in the whole heart (21) may obscure the important ventricle-specific changes in the putrescine/ spermidine ratio reported herewithin (Fig. 8) during preferential LV growth after birth. After birth the high fetal putrescine/ spermidine ratio is maintained in the $\mathrm{LV}$, but rapidly falls in the RV (Fig. 8), in part due to the higher LV ODC activity (Fig. 3). Correlating these changes with the histologic evidence of preferential LV myocyte hyperplasia in neonatal rat hearts (2) it may be speculated that a high putrescine/spermidine ratio is important for LV hyperplasia to continue after birth perhaps in response to the increased LV afterload (19). In contrast, RV afterload de- creases, RV myocytes do not undergo significant hyperplasia after birth (2), and putrescine/spermidine ratio falls. Subsequent myocyte growth by increase in cell size (hypertrophy) after day 7 may not be associated with an elevated putrescine/spermidine ratio. It is of interest to note that the putrescine/spermidine ratio increases from 0.35 to $0.9424 \mathrm{~h}$ in the adult rabbit RV following pulmonary artery banding (20); however, the acutely afterloaded LV in the rat did not result in an altered putrescine/spermidine ratio (7). These results suggest that at least in the rabbit acute changes in afterload may result in changes in polyamine metabolism such that there is an increase in putrescine to spermidine ratio. Future studies will be necessary to identify in which population of cells of the myocardium is polyamine synthesis changing with growth stimulation to further speculate on their role in myocardial growth (17).

This investigation has characterized the differential growth of the neonatal rabbit $\mathrm{LV}$ that may be in response to changes in hemodynamic workload. The initiation of the LV growth is accompanied by and possibly requires an increase in ODC specific activity and in putrescine synthesis. There also appears to be a transient increase in myocardial spermidine and spermine synthesis in both the developing $L V$ and the RV. The ratio of putrescine to spermidine increases in the $\mathrm{LV}$ and decreases in the RV immediately after birth and may be a characteristic polyamine profile of the rabbit ventricle following growth stimulation secondary to an acute change in afterload.

\section{REFERENCES AND NOTES}

1. Anderson, T. R. and Schanberg, S. M.: Ornithine decarboxylase activity in developing rat brain; J. Neurochem., 19: 1471 (1972).

2. Anversa, P., Olivetti, G., and Loud, A. V.: Morphometric study of early postnata development in the left and right ventricular myocardium of the rat. Circulation Res., 46: 495 (1980).

3. Bareis, D. L. and Slotkin, T. A.: Responses of heart ornithine decarboxylase and adrenal catehcholamines to methadone and sympathetic stimulants in developing and adult rats. J. Pharm. Exper. Thera., 205: 164 (1977).

4. Caldarera, C. M., Orlandini, G., Casti, A., and Moruzzi, G.: Polyamine and nucleic acid metabolism in myocardial hypertrophy of the overloaded rat heart. J. Mol. Cell. Cardiol., 6: 95 (1974).

5. Caldarera, C. M., Casti, A., Guarnieri, C., and Moruzzi, G.: Regulation of ribonucleic acid synthesis by polyamines. Biochem. J., 152: 91 (1975).

6. Casti, A., Guarnieri, C., Dall'Asta, R., and Clo, C.: Effect of spermine on acetylation of histones in rabbit heart. J. Mol. Cell. Cardiol., 9: 63 (1977).

7. Feldman, M. J. and Russell, D. J.: Polyamine biogenesis in left ventricle of the rat heart after aortic constriction. Amer. J. of Physiol., 5: 1199 (1972).

8. Friedman, W. F., Pool, P. E., Jacobivits, D., Seagren, S. C., and Braunwald, E.: Sympathetic innervation of the developing rabbit heart. Circulation Res., 23: 25 (1968).

9. Gibson, K. and Harris, P.: The in vitro and in vivo effects of polyamines on cardiac protein biosynthesis. Cardiovas. Res., 8: 668 (1974).

10. Hollenberg, M., Honbo, N., and Samorodin, A.: Cardiac cellular responses to altered nutrition in the neonatal rat. Amer. J. Physiol., 233: H356 (1977).

11. Lau, C. and Slotkin, T. A.: Regulation of rat heart ornithine decarboxylase: change in affinity for ornithine evoked by neuronal, hormonal, and ontogenetic stimuli. Mol. Pharmacol., 16: 504 (1979).

12. Lee, J. C., Taylor, J. F. N., and Downing, S. E.: A comparison of ventricular weights and geometry in newborn, young, and adult mammals. J. of App1. Physiol., 38: 147 (1975).

13. Lowry, O. H., Rosenbrough, N. J., Farr, A. L., and Randall, R. J.: Protein measurement with the folin phenol reagent. J. Biol. Chem., 193: 265 (1951).

14. Matshushita, S., Sogani, R. K., and Raben, M. S.: Ornithine decarboxylase in cardiac hypertrophy in the rat. Circ. Res., 31: 699 (1972).

15. Morgunov, N. and Liew, C. C.: Effects of spermine on acetylation of nucleohistone in the isolated perfused rat heart. J. Mol. Cell Cardiol., 9: 255 (1977)

16. Olivetti, G., Anversa, P., and Loud, A. V.: Morphometric study of early postnatal development in the left and right ventricular myocardium of the rat. Circulation Res., 46: 503 (1980).

17. Pegg, A. E. and Hibasami, H.: Rat polyamine metabolism during thyroxine induced cardiac hypertrophy. Amer. J. Physio., 239: E-372 (1980).

18. Riemenschneider, T. A., Ruttenberg, H. D., and Adams, F. H.: Maturational changes in left and right ventricular electromechanical intervals in the newborn lambs. Cardiovascular Res., 12: 228 (1978).

19. Rudolf, A. M.: In: Congenital Diseases of the Heart, pp 1-48 (Year Book Med. Publ., Chicago, I1l., 1974).

20. Russell, D. H., Shiverick, K. T., Hamrell, B. B., and Albert, N. R.: Polyamine synthesis during initial phases of stress-induced cardiac hypertrophy in rabbits. Amer. J. Physiol., 221: 1287 (1972).

21. Russell, D. H. and McVicker, T. A.: Polyamines in the developing rat and in supportive tissues. Biochim. Biophys. Acta, 259: (1972). 
22. Seiler, N., Knodgen, B., and Eisenbeiss, F.: Determination of di- and polyamines by high performance liquid chromatographic separation of their 5-dimethylaminonaphthalene-1-sulfonyl derivatives; J. of Chromatogr., 145: 29 (1978).

23. Smith, H. E. and Page, E.: Ultrastructural changes in rabbit heart mitochondria during the perinatal period. Develop. Biol., 57: 109 (1977).

24. Stastny, M. and Cohen, S.: Epiderman growth factor IV. The induction of ornithine decarboxylase. Biochim. Biophys. Acta, 204: 578 (1970).

25. Thadani, P. V. and Schanberg, S. M.: Effect of stress and sympathetic activity on adult rat cardiac and aortic ornithine decarboxylase activity. Life Sci., 25: 1009 (1979).

26. Williams-Ashman, H. G. and Canellakis, Z. N.: Polyamines in mammalian biology and medicine. Perspectives in Biology and Medicine, 23: 421 (1979).

27. Winick, M. and Noble, A.: Quantitative changes in DNA, RNA, and protein during prenatal and postnatal growth in the rat. Develop. Biol., 12: 451 (1965).

28. Requests for reprints should be addressed to: Dr. Robert J. Boucek, Jr., Department of Pediatrics and Biochemistry, Vanderbilt Medical Center, Nashville, TN 37232.

29. This research was supported in part by U.S. Public Health Service Research Grant (HL-16367) from the National Heart, Lung, and Blood Institute and Biomedical Science Research Grant \#RR-05424.

30. Received for publication March 17, 1981.

31. Accepted for publication October 1, 1981 .

\title{
PRESENTING THE BULLETIN OF THE INTERNATIONAL PEDIATRIC ASSOCIATION
}

\author{
THE BULLETIN OF THE INTERNATIONAL PEDIATRIC ASSOCIATION \\ IS NOT JUST ANOTHER PEDIATRIC JOURNAL
}

\section{READ THE BULLETIN OF THE IPA}

- Know more about what is happening in pediatrics in the world today.

- Enjoy papers in the "Special Contribution" section of the Bulletin by some of the most eminent pediatricians of our times.

- Gain an international perspective on matters of interest to you as a pediatrician.

- Keep abreast of IPA seminars, symposia and workshops on topics of the utmost importance to your profession.

- Learn when future international and regional pediatric meetings will be held and where to write for information.

Published quarterly in English, French and Spanish, the official languages of the International Pediatric Association, the Bulletin of the IPA provides a forum for the views of pediatricians from all over the world and an opportunity for you to know your colleagues in other countries.

\section{SUBSCRIBE TO THE IPA BULLETIN}

To receive more information concerning the Bulletin of the IPA please write to your national pediatric society or to the Managing Editor of the Bulletin at the address which follows:

Bulletin of the IPA

P.K. 2 Maltepe

Ankara, Turkey 